\title{
Relativistic Completion of Schrodinger's Quantum Mechanics by the Ether Theory
}

\author{
David Zareski ${ }^{1}$ \\ ${ }^{1}$ I.A.I, Israel Aerospace Industries Yehud, Israel \\ Correspondence: David Zareski, I.A.I, Israel Aerospace Industries Yehud, Israel. E-mail: zareski@inter.net.il
}

Received: June 10, 2019

Accepted: June 25, 2019

Online Published: July 15, 2019

doi:10.5539/apr.v11n4p52

URL: http://dx.doi.org/10.5539/apr.v11n4p52

\begin{abstract}
One recalls that we have shown in our precedent publications that the ether is an elastic isotropic medium. One presents the exact equation and its non-relativistic approximation that govern the ether in presence of a Schwarzschild-Coulomb field to which is submitted a $\operatorname{Par}(m, e)$ (particle of mass $m$ and of electric charge $e$ ). We present the exact relativistic solution of this exact equation in the circular case. We prove that the Schrödinger equation is such a non-relativistic approximation, that is, is a particular case of the ether elasticity theory.

One recalls that the Schrödinger equation was obtained by the use of operators and not from the theory of elasticity. It follows that this manner of obtaining this equation from operators is arbitrary and does not permit to obtain its complete relativistic form, but permits to reach absurd conclusions as, e.g., the cat that, at the same moment, is alive and dead. One shows then that other results ensuing from the Schrödinger equation are particular cases of the non-relativistic equation that governs the elastic ether, like for example: the Bohr-Sommerfeld condition, and the eigenstates function equation.

Résumé. Nous rappelons que nous avons montré dans nos publications précédentes que l'éther est un milieu élastique et isotrope. Nous présentons l'équation exacte et son approximation non-relativiste qui régit l'éther en présence d'un champ de Schwarzschild-Coulomb auquel est soumise une $\operatorname{Par}(m, e)$ (particule de masse $m$ et de charge électrique $e$ ). Nous présentons la solution relativiste exacte de cette équation exacte dans le cas circulaire. Nous démontrons que l'équation de Schrödinger est une telle approximation non-relativiste, c'est-àdire, est un cas particulier de la théorie de l'élasticité de l'éther.

Nous rappelons que l'équation que Schrödinger a été obtenue par l'utilisation d'opérateurs et non pas de la théorie de l'élasticité. Il s'ensuit que cette façon d'obtenir cette équation à partir d'opérateurs est arbitraire et ne permet pas d'obtenir l'équation complète relativiste de la mécanique quantique, mais permet d'arriver à des conclusions absurdes comme, e.g., le chat qui est mort et vivant au même instant. Nous montrons de plus que d'autres résultats découlant de l'équation de Schrödinger sont des cas particuliers de l'équation non-relativiste qui gouverne l'éther élastique, comme par exemple: la condition de Bohr-Sommerfeld, et l'équation aux états propres.
\end{abstract}

Keywords: Quantum Mechanics and Ether Elasticity, Generalization of Schrödinger's Equation, Physical Signification of the Quantum Effects

\section{Introduction}

We denote by $p_{\mu}$ the covariant components of the momentum tensor, by $x^{\mu}$ the contra-variant coordinates, by $\dot{x}^{\mu}$ the time derivative of $x^{\mu}$, i.e., $\dot{x}^{\mu} \equiv d x^{\mu} / d t$, by $\partial_{\mu}$ the partial derivatives $\partial / \partial x^{\mu}$ and by $\operatorname{Par}(m, e)$ the particle of mass $m$ and electric charge $e$.

One recalls that Schrödinger's equation was obtained by the use of operators inspired from the de Broglie wave $\psi_{B}$ for which the expression is

$$
\psi_{B}=\left|\psi_{B}\right| \exp \left[-(i / \hbar)\left(W_{k i n} t-p \boldsymbol{x}\right)\right]
$$

This wave $\psi_{B}$ of constant amplitude $\left|\psi_{B}\right|$, is associated to a free particle of mass $\mathrm{m}$, of constant velocity $V$ along the coordinate $x$, of constant spatial momentum vector $p$, and of constant kinetic energy $W_{\text {kin }}$. It appears that if to $W_{k i n}$ is associated the operator $i \hbar \partial_{t}$ and to $p \equiv m V$, the operator $-i \hbar \partial_{x}$, that is, to $m V^{2} / 2$, is associated the operator $-\left[\hbar^{2} /(2 m)\right] \partial_{x x}$, then $\psi_{B}$ is the solution of the differential equation

$$
-\left[\hbar^{2} /(2 m)\right] \partial_{x x} \psi_{B}=i \hbar \partial_{t} \psi_{B}
$$


Now, Schrödinger introduced the operators inspired from those here above defined, i.e., $-i \hbar \partial_{j}$ and $i \hbar \partial_{t}$ and considered the case where the particle is submitted in addition, to a static potential of forces $U$. In this case, if $W$ denotes the total energy other than the mass energy, then to the elements of the relation

$$
m V^{2} / 2+U=W
$$

he assigned the operators here above defined, and obtained the following so called Schrödinger equation:

$$
-\left(\hbar^{2} / 2 m\right) \nabla^{2} \psi+U \psi=i \hbar \partial_{t} \psi
$$

In the present paper we show that Equation (4) is a non-relativistic particular form of the general equation that governs the ether. Therefore, the theory of the ether permits, as we show here below, to generalize this equation to the relativistic case, i.e., to the exact form. In that context one recalls in particular that Maxwell and Einstein presumed the existence of this ether. Indeed, Maxwell wrote in Art. 866 (Maxwell, 1954):

"Hence all these theories lead to the conception of a medium in which the propagation takes place, and if we admit this medium as an hypothesis, I think it ought to occupy a prominent place in our investigations."

and Einstein (1920):

"Recapitulating, we may say that according to the general theory of relativity space is endowed with physical qualities; in this sense, therefore, there exists an ether. According to the general theory of elativity space without ether is unthinkable; for in such space there not only would be no propagation of light, but also no possibility of existence for standards of space and time (measuring-rods and clocks), nor therefore any space-time intervals in the physical sense."

As shown in Zareski $(2001,2012,2013)$ the "ether elasticity equation" that governs the displacements $\xi$ of the points of the ether ensues from the Navier-Stokes-Durand equation of elasticity, that takes also into account the densities of couples applied to the elastic medium. Theses densities of couples that play a important role in the physcs of the ether since they complete the Navier-Stokes equation of elasticity, were introduced by E. Durand (1953).

The ether elasticity equations are presented in Equations (30)-(32) of Zareski (2013) and in Equations (4.4)-(4.6) of Zareski (2015). In the present paper one considers these equations in the case where the field of forces that act on a $\operatorname{Par}(m, e)$ is created by an immobile $\operatorname{Par}\left(m_{0}, q_{0}\right)$, i.e., where, as we show, the changes $\xi$ of the points of the ether due to the motion of the $\operatorname{Par}(m, e)$ under the influence of $\operatorname{Par}\left(m_{0}, q_{0}\right)$ and also due to the $\operatorname{Par}\left(m_{0}, q_{0}\right)$ itself, are sums of waves that propagates in the ether. It appears that as we show in the present paper that in this case the ether elasticity theory generalizes the quantum mechanics since it yields as we show, not only the Schrödinger equation, which is, as we show, a non-relativistic approximation, but also the relativistic complete equation i.e., the exact wave equation associated to a particle in the Schwarzschild- Coulomb fields due to a $\operatorname{Par}\left(m_{0}, q_{0}\right)$ immobile at the origin $O$ of the coordinates. This new equation ensues, as we show, from the Navier-Stokes-Durand equation.

As shown in our previous publication Zareski (2014), these waves $\xi$ are called "particle waves" because they are associated to the $\operatorname{Par}(m, e) s$ as following: An adequate superposition $\hat{\xi}$ of waves $\xi$ forms a globule that moves like a $\operatorname{Par}(m, e)$ and possesses all the physical properties of the $\operatorname{Par}(m, e) s$, therefore a $\operatorname{Par}(m, e)$ can be considered as such a globule, in particular a $\operatorname{Par}(0,0)$ is a photon and the waves $\xi$ associated to it are electromagnetic.

Definition. We call Schwarzschild-Coulomb field, the field created by a particle of mass $m_{0}$ and of electrical charge $q_{0}$, i.e., by a $\operatorname{Par}\left(m_{0}, q_{0}\right)$, immobile at the origin $\boldsymbol{O}$ of the coordinates.

\section{Equation Governing the Ether in Presence of a Schwarzschild-Coulomb Field Acting on a Massive and Electricaly Charged Particle}

\subsection{The Relativistic Exact Case}

The particle wave $\xi$ associated to a $\operatorname{Par}(m, e)$ is, Cf. Sec. V of Zareski (2013), and Equation (4.5) of Zareski (2015), the solution of the following ether elasticity wave equation:

$$
\operatorname{curl}\left(V_{P}^{2} \operatorname{curl} \xi\right)=\omega^{2} \xi
$$

where $V_{P}$ denotes the phase velocity of $\xi$, and $\omega$ a constant pulsation. A $\operatorname{Par}\left(m_{0}, q_{0}\right)$ immobile at $\boldsymbol{O}$ creates a Schwarzschild-Coulomb field. The expression for $V_{P}$ of $\xi$ associated to a $\operatorname{Par}(m, e)$ submitted to such a field is (Zareski, 2012, 2013, 2015), 


$$
V_{P}^{2}=\frac{c^{2} \gamma^{2} E_{T}^{2}}{\gamma_{b}^{2}\left[\left(E_{T}+e A_{4}\right)^{2}-\gamma^{2}\left(m c^{2}\right)^{2}\right]}
$$

Where

$$
\begin{gathered}
\gamma^{2} \equiv 1-\alpha / r \\
\alpha \equiv 2 m_{0} k / c^{2} \\
\gamma_{b}{ }^{2} \equiv 1+\alpha\left(\cos ^{2} b\right) /\left(r \gamma^{2}\right) \\
e A_{4} \equiv-\left|e q_{0}\right| /\left(4 \pi \varepsilon_{0} r\right)
\end{gathered}
$$

where " $b$ " denotes the angle made by the radius vector $\boldsymbol{r}$ and the trajectory element of the $\operatorname{Par}(m, e), k$, the constant of gravitation, and $E_{T}$, the total particle energy, for which the expression is

$$
E_{T} \equiv m c^{2}+h v \equiv \hbar \omega
$$

In this expression $h v$ denotes the energy of $\operatorname{Par}(m, e)$ other than the mass energy, $h$ the Planck constant and $v$ a frequency. Note that $E_{T}$ is constant since the fields to which it is submitted are static that is $v$ and therefore $\omega$ are constant. Furthermore, one recalls that the velocity $V$ of a $\operatorname{Par}(m, e)$ is related to the phase velocity $V_{P}$ of the wave associated to this particle by Zareski (2013), the relation

$$
\frac{\partial}{\partial E_{T}}\left(\frac{E_{T}}{V_{P}}\right)=\frac{1}{V}
$$

That is to say that, from (6) and (12) it follows that the expression for the particle velocity $V$ is

$$
V=\frac{c \gamma}{\gamma_{a}} \sqrt{1-\left(\frac{\gamma m c^{2}}{E_{T}+e A_{4}}\right)^{2}}
$$

We present now Equation (5) in a more specific form, indeed, this Equation (5) can be also written as following:

$$
\operatorname{curl}(\operatorname{curl} \xi)+\operatorname{grad}\left(\log V_{P}{ }^{2}\right) \wedge \operatorname{curl} \xi=\left(\omega^{2} / V_{P}{ }^{2}\right) \xi
$$

that is also:

$$
-\nabla^{2} \xi+\operatorname{grad}(\operatorname{div} \xi)+\operatorname{grad}\left(\log {V_{P}}^{2}\right) \wedge \operatorname{curl} \xi=\left(\omega^{2} / V_{P}^{2}\right) \xi
$$

We show now that Equation (15) can be simplified because one can take

$$
\operatorname{div} \xi=\mathbf{0}
$$

indeed,

$$
\operatorname{div} \boldsymbol{B}=0 \text { and } \boldsymbol{B} \equiv \rho_{0} \partial_{t} \xi
$$

Cf. Equation (34) of Zareski (2013), and Sec. V of Zareski (2015), it follows that Equation (16), implies that Equation (15) becomes:

$$
-\nabla^{2} \xi+\operatorname{grad}\left(\log V_{P}^{2}\right) \wedge \operatorname{curl} \xi=\left(\omega^{2} / V_{P}^{2}\right) \xi
$$

Equation (18) is the other form that takes Equation (5) that governs the ether influenced by the field created by a $\operatorname{Par}\left(m_{0}, q_{0}\right)$ located at $\boldsymbol{O}$ and by a $\operatorname{Par}(m, e)$ submitted to this field.

\subsection{Recall on the Non-Relativistic Case}

In the non-relativistic approximation, $\alpha / r$ is neglected in front of 1 , and $h v+e A_{4}$ in front of $m c^{2}$, but $\left(m c^{2}\right)^{2} \alpha / r$ is not neglected. With these non-relativistic approximations, one has

$$
\left.\left(E_{T}+e A_{4}\right)^{2}-\gamma^{2}\left(m c^{2}\right)^{2} \cong 2 m c^{2}[h v+\hat{a} / r]\right]
$$

where $\hat{a}$ is defined by

$$
\hat{a} \equiv\left|e q_{0}\right| /(4 \pi \varepsilon)+m m_{0} k
$$

and $V_{P}^{2}$ defined in (6), becomes

$$
V_{P}{ }^{2} \cong{E_{T}}^{2} /[2 m(h v+\hat{a} / r)]
$$


Furthermore, as proven in Zareski (2015), in this non-relativistic approximation, the quantity $\boldsymbol{g r a d}\left(\log V_{P}^{2}\right) \wedge$ $\operatorname{curl\xi }$ is negligible in front of $\frac{2 m}{\hbar^{2}}\left(\frac{\hat{a}}{r}+h v\right)$. Finally, with these non-relativistic approximations, Equation (18) becomes

$$
-\frac{\hbar^{2}}{2 m} \nabla^{2} \xi_{r} \cong\left(\frac{\hat{a}}{r}+h v\right) \xi_{r}
$$

Let us consider again Equation (22), in this equation one can write that $\xi_{r}$ is the product of a function $\zeta(r)$ that depends only upon $r$ and of the function $\exp (-i 2 \pi v t)$, that is

$$
\xi_{r} \equiv \zeta(r) \exp (-i 2 \pi v t)
$$

In this case Equation (22) becomes

$$
-\frac{\hbar^{2}}{2 m} \nabla^{2} \xi_{r} \cong \frac{\hat{a}}{r} \xi_{r}+i \hbar \partial_{t} \xi_{r}
$$

indeed considering (23), one can verify

$$
i \hbar \partial_{t} \xi_{r}=h v \xi_{r}
$$

Equation (24) is the same as Equation (4) obtained by Schrödinger by the use of operators as shown in Sec. 1, here above and, as we demonstrated, is also the non-relativistic approximation of Equation (5) that governs the ether submitted to the Schwarzschild-Coulomb field, therefore:

The ether elasticity theory includes the exact relativistic quantum mechanics equation that generalizes the Schrödinger quantum mechanics equation which is the non-relativistic approximation of this exact quantum mechanics equation.

Definition. We call "relativistic circular case", the case where the $\operatorname{Par}(\mathrm{m}, \mathrm{e})$ under the influence of a $\operatorname{Par}\left(\mathrm{m}_{0}, \mathrm{q}_{0}\right)$ is associated to circularly trajectories centered at 0 .

\section{Example: The Circular Case}

\subsection{The Relativistic Exact Circular Case}

In a bound state of a $\operatorname{Par}(m, e)$ submitted to a Schwarzschild- Coulomb field due to $\operatorname{Par}\left(m_{0}, q_{0}\right)$, located at the origin $\mathrm{O}$, the waves $\xi$ interfere with themselves. In these interferences, only remind certain waves $\xi$ that do not destruct themselves in these interferences, but on the contrary, resonates. Such resonance happen when such close trajectory of $\operatorname{Par}(m, e)$ contains an integer number " $n$ " of wave lengths $I_{w}$. Let us consider the case where the trajectories described by $\operatorname{Par}(m, e)$ attracted by $\operatorname{Par}\left(m_{0}, q_{0}\right)$ are circular, and located in the plane $\theta=\pi / 2$. In such a circular state, $I_{w}$ is constant since it does not depend upon the angle $\phi$ described by the constant radius vector $r$ denoted then $\rho$. The resonance condition happens when

$$
2 \pi \rho / I_{w}=n
$$

Now the expression for the total frequency $v_{T}$ is

$$
v_{T}=E_{T} / h
$$

and since $I_{w}=V_{P} / v_{T}$, the expression for $I_{w}$ is

$$
I_{w}=h V_{P} / E_{T}
$$

In this circular case, we can express the component $p_{\phi}$ of the momentum tensor, it is defined by

$$
p_{\phi} \equiv \partial L_{G c} / \partial \dot{\phi}
$$

$w$ here $L_{G c}$ is the Lagrange-Einstein function in this case. Indeed, in this case where the trajectories are circumferences of radius $\rho$ in the plane $\theta=\pi / 2$, the expression for $L_{G c}$ is

$$
L_{G c}=-m c \dot{s}_{c}+e A_{4} \dot{x}^{4} / c
$$

where $\dot{s}_{c}$ is the value of $\dot{s}$ on this circle, for which the value is: Cf. Equations (3.65) and (7.38) of Zareski (2015), 


$$
\dot{s}_{c}=\sqrt{\gamma^{2} c^{2}-\dot{\phi}^{2} \rho^{2}}
$$

Now, since on the circle of radius $\rho$, one has

$$
\dot{\phi} \rho=V
$$

it follows that

$$
\dot{s}_{c}=\sqrt{\gamma^{2} c^{2}-V^{2}}
$$

From (30) and (29), one has

$$
p_{\phi}=m c \rho V / \dot{s}_{c}
$$

where $V$ is given by (13). Now one imposes the Bohr-Sommerfeld condition

$$
p_{\phi}=n \hbar
$$

where $\dot{s}_{c}$ is given in (33), then,

$$
n \hbar=m c \rho V / \sqrt{\gamma^{2} c^{2}-V^{2}}
$$

Taking the explicit expression (13) for $V$ into account, one obtains

$$
n \hbar=[\rho /(\gamma c)] \sqrt{\left(E_{T}+e A_{4}\right)^{2}-\left(\gamma m c^{2}\right)^{2}}
$$

For a given $n \hbar$, the unknowns are $E_{T}$, (i.e., $v_{T}$ ), and $\rho$. A first equation of these unknowns is Equation (37). In order to determine a second equation of these unknowns $E_{T}$ and of $\rho$, we use the motion equation

$$
\frac{d}{d t} \frac{\partial L_{G c}}{\partial \dot{r}}-\frac{\partial L_{G c}}{\partial r}=0
$$

Now, considering (30), one sees that $\partial L_{G c} / \partial \dot{r}=0$, it follows that $\partial L_{G c} / \partial r=0$, therefore (38) becomes,

$$
-\frac{1}{2} m c\left(\alpha c^{2} / \rho^{2}-2 V^{2} / \rho\right)+e \partial_{\rho} A_{4} \sqrt{\gamma^{2} c^{2}-V^{2}}=0
$$

where, on the circle, Equation (13) becomes:

$$
V^{2}=(c \gamma)^{2}\left[1-\left(\frac{\gamma m c^{2}}{E_{T}+e A_{4}}\right)^{2}\right]
$$

since $\gamma_{b}=1$. Now, Equations (39) and (37), are fundamental, indeed, from Equation (37), one deduces

$$
\left(E_{T}+e A_{4}\right)^{2}=(n \hbar \gamma c / \rho)^{2}+\left(\gamma m c^{2}\right)^{2}
$$

introducing this expression for $\left(E_{T}+e A_{4}\right)^{2}$ into (40), and denoting then $V^{2}$ by $V_{n \hbar}{ }^{2}$, one obtains

$$
V_{n \hbar}^{2}=(c \gamma)^{2}\left[\frac{(n \hbar \gamma c / \rho)^{2}}{(n \hbar \gamma c / \rho)^{2}+\left(\gamma m c^{2}\right)^{2}}\right]
$$

Since $V^{2}$ becomes $V_{n \hbar}{ }^{2}$, it follows that (39) becomes

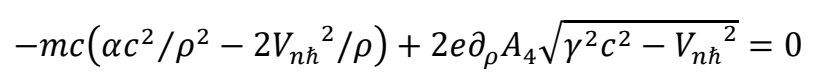

From Equation (43), one deduces the radius $\rho$ described by the electron as a function of $n \hbar$, then for each known value of $\rho$, one deduces the value of $E_{T}$, i.e. of $v_{T}$ from Equation (41).

\subsection{Recall on the Non-Relativistic Circular Case}

We determine now the values of $v$ and $\rho$ that cause this resonance by determining two equations involving them. Now, in this space-time, the expression for the velocity $V$ is given in Equation (13), which, in the non-relativistic approximation, becomes considering (19),

$$
V \cong \sqrt{\frac{2}{m}\left(h v+\frac{\hat{a}}{\rho}\right)}
$$


It follows, by inserting (44) in (34), that

$$
p_{\phi} \cong \rho \sqrt{2 m(h v+\hat{a} / \rho)}
$$

i.e.,

$$
(n \hbar)^{2} \cong 2 m \rho^{2}(h v+\hat{a} / \rho)
$$

Eq. (46) yields on account of (44),

$$
V^{2} \cong[n \hbar /(\rho m)]^{2}
$$

Keeping in mind that $\dot{\phi} \rho=V$, and that $\partial A_{4} / \partial \rho=q_{0} /\left(4 \pi \varepsilon_{0} \rho^{2}\right)$, (39) becomes in the non-relativistic approximation,

$$
m \rho V^{2}-\hat{a} \cong 0
$$

and taking (47) and (20) into account, one gets

$$
\rho \cong(n \hbar)^{2} /(\hat{a} m)
$$

Inserting (49) into (46) one obtains

$$
h v=-(m / 2)[\hat{a} /(n \hbar)]^{2}
$$

and since $\left|e q_{0}\right| /(4 \pi \varepsilon)>>m m_{0} k$, Equation (50) can be written with a good approximation

$$
h v \cong-(m / 2)\left[e q_{0} /(4 \pi \varepsilon n \hbar)\right]^{2}
$$

One sees that the relations (51) and (49), obtained from the theory of the waves $\xi$ in the elastic ether are the same as those obtained from the current quantum mechanics theory. It follows again that there is a relation between these two theories and, moreover, that the ether elasticity theory generalizes the usual quantum mechanics theory. Furthermore, one sees that, taking Equation (53) into account, Equation (22) becomes for the circular trajectory of the electron, the following eigenstates equation

$$
-\frac{\hbar^{2}}{2 m} \nabla^{2} \xi_{r}-\frac{\hat{a}}{r} \xi_{r} \cong-\frac{1}{2} \frac{\hat{a}^{2} m}{(n \hbar)^{2}} \xi_{r}
$$

for which the solutions that are quantum states, are given, e.g., in Durand (1970), Chap. XI.

\section{Conclusions}

The wave equation associated to a particle, for example submitted to a Schwarzschild-Coulomb field, was obtained by two completely different reasonings.

The first reasoning was Schrödinger's, he associated operators to physical quantities inspired from the de Broglie plane wave, for example, to the total energy, he associated the operator $i \hbar \partial_{t}$, to the momentum tensor, the operator $-i \hbar \partial_{j}$, etc. He obtained then the so-called Schrödinger equation.

In the second reasoning, we demonstrated that, from the equation governing the ether, one obtains the relativistic exact wave equations associated to the particle in this field, and also its non-relativistic approximation which appears to be the same as Schrödinger's equation.

Now, Schrödinger's method of obtaining this non-relativistic equation governing the wave associated to a particle appears to be artificial since it is based on the use of operators inspired from the de Broglie's plane wave for example: he did not consider the general relativity and brought unlogic conclusions like, for example, the cat that can be alive and dead at the same instant.

We showed that the quantum mechanics equation obtained by Schrödinger's method using the operators is only a particular case of the ether elasticity theory. Indeed, we showed here above that the fundamental Equation (5) that rules the ether, takes, in a particular case, the particular relativistic form (18). In the non-relativistic case, this equation takes the form (22) which is the same as the equation developed by Schrödinger, but the manner of obtaining this equation, presented in the present paper, i.e., obtained from the fundamental Equation (5) that rules the ether, ensues from a logical process that permits to obtain the exact equation, i.e., that take into account the theory of relativity.

\section{Conflict of interests}

The authors declare that there is no conflict of interests regarding the publication of this paper. 


\section{References}

Durand, E. (1953). Electrostatique et Magnétostatique. Masson \& Cie.

Durand, E. (1970). Mécanique Quantique (Tome 1). Masson \& Cie.

Einstein, A. (1920). Ether and the theory of relativity. The collected papers of A. Einstein (Springer, Berlin, 1920), address delivered on May 5, 1920, at the University of Leyden.

Maxwell, J. C. (1954). A Treatise on Electricity and Magnetism. Dover, New-York.

Zareski, D. (2001). The elastic interpretation of electrodynamics. Phys. Let., 14(5).

Zareski, D. (2012). On the elasto-undulatory interpretation of fields and particles. Physics Essays Publications, 25 , 2.

Zareski, D. (2013). Field and wave-particle reciprocity as changes in an elastic medium: The ether. Physics Essays Publications, 26, 2.

Zareski, D. (2014). The quantum mechanics as also a case of the ether elasticity theory. Appl. Phys. Research, $6(4)$.

Zareski, D. (2015). Unification of Physics by the Ether Elasticity Theory. LAP LAMBERT Academic Publishing.

\section{Copyrights}

Copyright for this article is retained by the author(s), with first publication rights granted to the journal.

This is an open-access article distributed under the terms and conditions of the Creative Commons Attribution license (http://creativecommons.org/licenses/by/4.0/). 